substantially just by changing from the TLC to the TLI. Therefore, we regard our results as reliable.

Furthermore, Palaniyappan criticises the factor analysis of the PANSS in the small patient samples used in neuroimaging studies. He is right that a factor analytic approach in such small samples is critical if the patients group is randomly selected. In order to be able to study formal thought disorder with neuroimaging techniques, we recruited a specifically selected patient sample, which mainly differs in formal thought disorder severity and barely in other psychopathological categories. To obtain such a selected sample, the recruitment took years. Patients were matched as closely as possible for all other psychopathology traits in addition to the items of formal thought disorder of PANSS. The factor analysis and the correlation of the factors with the severity of formal thought disorder were only used to document this special patient selection. Therefore, it is no surprise that the factors of our factor analysis do not match the factors of unselected samples of patients with schizophrenia. ${ }^{3}$ To study specific psychopathological phenomena like formal thought disorder by means of neuroimaging, such a specific selection of patients is necessary to extract reliable results. In our patient group we did not observe any significant correlation between formal thought disorder and (a) positive symptoms (without PANSS item P2): $r=0.39, P=0.19$; and (b) negative symptoms (without PANSS item N5): $r=0.04, P=0.90$. We therefore can conclude that our results are due to positive or negative symptoms in general.

Concerning the issue of the sample size in neuroimaging studies, our results were corrected for multiple comparisons. This approach is a common and accepted way to handle this problem in neuroimaging studies in general.

1 Liddle PF, Ngan ETC, Caissie SL, Anderson CM, Bates AT, Quested DJ, et al. Thought and Language Index: an instrument for assessing thought and language in schizophrenia. Br J Psychiatry 2002; 181: 326-30.

2 Andreasen NC, Grove W. Thought, language and communication in schizophrenia: diagnosis and prognosis. Schizophr Bull 1986; 12: 348-59.

3 Fitzgerald PB, de Castella AR, Brewer K, Filia K, Collins J, Davey P, et al. A confirmatory factor analytic evaluation of the pentagonal PANSS model. Schizophr Res 2003; 61: 97-104.

Helge Horn, University Hospital of Psychiatry, University of Bern, Switzerland. Email: horn@puk.unibe.ch; Andrea Federspiel, University Hospital of Psychiatry, University of Bern, Switzerland

doi: 10.1192/bjp.195.1.85b

\section{Public attitudes towards mental illness}

It is tempting to accept the conclusion from the paper by Mehta et $a l^{1}$ that the Scottish anti-stigma campaign 'see me' has successfully influenced public attitudes north of the border. However, it is not clear that this is an appropriate conclusion from the data they present.

They describe random sampling techniques whereby 2000 adults representative of the UK population were surveyed. One presumes that this would give rise to cohorts in England which were roughly ten times larger than those in Scotland. Comparing the year 2000 against 2003, they observed a deterioration for 17/25 stigma questionnaire items in England against only 4/25 in Scotland, and concluded that Scotland's dubious distinction of having done less badly suggested that 'see me' had been effective. Can they assure us that this difference did not arise simply because the much larger samples in England would be more likely to show a statistically significant difference than smaller Scottish samples?
1 Mehta N, Kassam A, Leese M, Butler G, Thornicroft G. Public attitudes towards people with mental illness in England and Scotland, 1994-2003. Br J Psychiatry 2009; 194: 278-84.

John M. Eagles, Royal Cornhill Hospital, Cornhill Road, Aberdeen AB25 2ZH, Scotland. Email: john.eagles@nhs.net

doi: 10.1192/bjp.195.1.86

Authors' reply: Professor Eagles writes to ask whether our conclusion from the paper is that the Scottish 'see me' anti-stigma campaign has positively influenced public attitudes about mental illness in Scotland. He suggests that this conclusion may be inappropriate because the populations in Scotland and England produce different sample sizes, given that the population of England is roughly ten times that of Scotland. We are very grateful to Professor Eagles for his comments as they allow us to provide some more information on these surveys than we could include in the original paper. ${ }^{1}$

As he rightly says, direct evidence of the position in Scotland vis-à-vis England is not provided by comparison of only those significant changes within the two sites. We had hoped to pursue this question further with analysis of future surveys, which would have given us power to make the comparisons between Scotland and England adequately, but unfortunately the wording of the Scottish survey has been changed so this will not be possible. The existing data-sets do, however, show limited evidence in favour of Scotland when comparing their respective mean changes from immediately pre- to post-campaign periods (2000 v. 2003). Of the 25 items, 6 differed between the sites at a nominal 0.1 significance level. One item (26: fear of downgrading residential areas) favoured England at $P=0.05$. The others favoured Scotland: items 7-9 at $P=0.1$ (to do with tolerance), and items 10 and 13 (the need to spend money and care for people with mental illness) at $P=0.05$. As we stated in our paper, the evidence may be consistent with an early positive effect of 'see me', but this possible association requires further investigation, although we accept that it is far from conclusive and needs further verification.

1 Mehta N, Kassam A, Leese M, Butler G, Thornicroft G. Public attitudes towards people with mental illness in England and Scotland, 1994-2003. Br J Psychiatry 2009; 194: 278-84.

Nisha Mehta, Health Service and Population Health Research Department, Institute of Psychiatry, King's College London, Box PO29 De Crespigny Park, London SE5 8AF, UK. Email: nisha.s.mehta@kcl.ac.uk; Aliya Kassam, Morven Leese, Georgia Butler, Graham Thornicroft, Health Service and Population Health Research Department, Institute of Psychiatry, King's College London, UK

doi: 10.1192/bjp.195.1.86a

\section{Is there core diffusion tensor imaging pathology in schizophrenia?}

Kanaan et al ${ }^{1}$ reported widespread abnormalities in white matter in 76 patients with schizophrenia compared with 76 healthy controls. A secondary analysis of 45 patients showed mean extracted fractional anisotropy scores to be unrelated to illness duration and duration of antipsychotic treatment. We wish to make two comments.

First, their main hypothesis that they would reconcile inconsistencies in the literature is a worthy, but elusive, goal. The problem of nosological heterogeneity 'afflicts' not only the definition of schizophrenia, but also the interpretation of fractional anisotropy localisation. Fractional anisotropy score localities are commonly cited in terms of grey or white matter terminology. Given such heterogeneity, it would suffice to adopt 
the lesser goal of showing core pathology (in other words, the Venn diagram intersection). Coreness of pathology allows for diversity, without having to reconcile everything.

Second, we note that the secondary analysis was performed to dissect out the effects of the disorder from that of illness duration and treatment. The authors achieved this by using fractional anisotropy scores extracted from the principal analysis, which were then used to compare chronically with briefly medicated patients. Perhaps newly diagnosed, antipsychotic-naive patients help most to partition out these effects ${ }^{2}$ but they are not essential. An alternative approach is voxel-based ANOVA covarying for illness duration and atypical antipsychotic duration/dosage since this can help maximise anatomical coverage (particularly in the striatum where antipsychotic effects are detectable at even 2-3 weeks of treatment ${ }^{3,4}$ ).

1 Kanaan R, Barker G, Brammer M, Giampietro V, Shergill S, Woolley J, et al. White matter microstructure in schizophrenia: effects of disorder, duration and medication. Br J Psychiatry 2009; 194: 236-42.

2 Cheung V, Cheung C, McAlonan GM, Deng Y, Wong JG, Yip L, et al. A diffusion tensor imaging study of structural dysconnectivity in nevermedicated, first-episode schizophrenia. Psychol Med 2008; 38: 877-85.

3 Szeszko PR, Ardekani BA, Ashtari M, Kumra S, Robinson DG, Sevy S, et al. White matter abnormalities in first-episode schizophrenia or schizoaffective disorder: a diffusion tensor imaging study. Am J Psychiatry 2005; 162: 602-5.

4 Chua SE, Deng Y, Chen EY, Law CW, Chiu CP, Cheung C, et al. Early striatal hypertrophy in first-episode psychosis within 3 weeks of initiating antipsychotic drug treatment. Psychol Med 2009; 39: 793-800.

Siew E. Chua, Department of Psychiatry, and State Key Laboratory for Cognitive Sciences, The University of Hong Kong, Hong Kong. Email: sechua@hku.hk; Grainne McAlonan, Department of Psychiatry, The University of Hong Kong, Hong Kong

doi: 10.1192/bjp.195.1.86b

Authors' reply: We agree that the process of establishing a definitive extent of white matter disruption in schizophrenia, and its relationship with illness duration and antipsychotic medication, is likely to be a lengthy one - larger studies such as ours notwithstanding. But we would like to clarify our reasoning with respect to Chua \& McAlonan's comments and the methodological alternatives they suggest.

First, we suspect that a 'core pathology' for white matter abnormalities in schizophrenia may be rather more elusive than the reconciliation we attempted. Although the recent metaanalysis by Ellison-Wright \& Bullmore ${ }^{1}$ has found areas of most common difference in the 15 studies they examined, it should be noted that only a fraction of the studies they looked at shared these differences - and the history of diffusion tensor imaging in schizophrenia is full of such conflicts. ${ }^{2}$ Although there may indeed be areas of greater difference, the evidence is against any difference that is common to all.

Second, with regard to distinguishing the effects of duration of illness and antipsychotic medication, drug-naive cohorts clearly offer enormous potential as the authors acknowledge. Such cohorts are difficult to obtain in high-income countries however, and the alternative approach they suggest - of ANOVA with covariation - has similar difficulties, since duration of treatment and illness will be so strongly correlated in most samples. We also note that the studies they cite as demonstrating the effectiveness of this approach either did not covary for medication exposure ${ }^{3}$ or did not use diffusion tensor imaging. ${ }^{4}$

1 Ellison-Wright I, Bullmore E. Meta-analysis of diffusion tensor imaging studies in schizophrenia. Schizophr Res 2009; 108: 3-10.

2 Kanaan RA, Kim JS, Kaufmann WE, Pearlson GD, Barker GJ, McGuire PK. Diffusion tensor imaging in schizophrenia. Biol Psychiatry 2005; 58: 921-9.

3 Szeszko PR, Ardekani BA, Ashtari M, Kumra S, Robinson DG, Sevy S, et al. White matter abnormalities in first-episode schizophrenia or schizoaffective disorder: a diffusion tensor imaging study. Am J Psychiatry 2005; 162: 602-5

4 Chua SE, Deng Y, Chen EY, Law CW, Chiu CP, Cheung C, et al. Early striatal hypertrophy in first-episode psychosis within 3 weeks of initiating antipsychotic drug treatment. Psychol Med 2009; 39: 793-800.

Richard A. Kanaan, Institute of Psychiatry, PO 62, Denmark Hill, London SE5 9RJ UK. Email: r.kanaan@iop.kcl.ac.uk; Gareth Barker, Philip McGuire, Institute of Psychiatry, King's College London, UK

doi: 10.1192/bjp.195.1.87

\section{Corrections}

Differential efficacy of escitalopram and nortriptyline on dimensional measures of depression. BJP, 194, 252-259. The two last-named authors, Dr K.J. Aitchison and Professor P. McGuffin, are the GENDEP Principal Investigators and contributed equally to the work. In addition, Dr Aitchison's qualifications include MRCPsych.

Caption for cover picture, April 2009. BJP, 194, A14. The text and image were submitted by Wojtek Wojcik; edited by Allan Beveridge. 